\title{
What Is the Ideal Entry Point for Transforaminal Endoscopic Lumbar Discectomy?
}

\author{
Jong Un Lee, ${ }^{1}$ Ki Jeoung Park, ${ }^{1}$ Ki Hong Kim, ${ }^{1}$ Man Kyu Choi, ${ }^{2}$ Young Hwan Lee, ${ }^{3}$ Dae-Hyun Kim \\ Department of Neurosurgery, Daegu Catholic University Medical Center, Catholic University of Daegu College of Medicine, Daegu, Korea \\ Department of Neurosurgery, Kyung Hee University Hospital, Kyung Hee University College of Medicine, Seoul, Korea \\ Department of Radiology, ${ }^{3}$ Daegu Catholic University Medical Center, Catholic University of Daegu College of Medicine, Daegu, Korea
}

Objective : The method of approach during transforaminal endoscopic lumbar discectomy (TELD) has been the subject of repeated study. However, the ideal entry point during TELD has not been studied in detail. Therefore, this study investigated the ideal entry point for avoiding complications using computed tomography (CT) scans obtained from patients in the prone position.

Methods : Using CT scans obtained from patients in the prone position, we checked for retroperitoneal or visceral violations and measured the angles of approach with five conventional approach lines drawn on axial CT scans at each disc space level (L2-3, L3-4, and $\mathrm{L} 4-5$ ). We also determined the ideal entry point distance and approach angles for avoiding retroperitoneal or visceral violations. Correlation analysis was performed to identify the patient characteristics related to the ideal entry point properties.

Results : We found that the far lateral approach at the L2-3 level resulted in high rates of visceral violation. However, rates of visceral violation at the L3-4 and L4-5 levels were remarkably low or absent. The ideal angles of approach decreased moving caudally along the spine, and the ideal entry point distances increased moving caudally along the spine. Weight, body mass index $(\mathrm{BMI})$, and the depth of the posterior vertebral line from the skin were positively associated with the distance of the ideal entry point from the midline.

Conclusion : We reviewed the risk of the extreme lateral approach by analyzing rates of retroperitoneal and visceral violations during well-known methods of approach. We suggested an ideal entry point at each level of the lumbar spine and found a positive correlation between the distance of the entry point to the midline and patient characteristics such as BMI, weight, and the depth of the posterior vertebral line from the skin.

Key Words : Endoscopy · Diskectomy · Percutaneous.

\section{INTRODUCTION}

Transforaminal endoscopic lumbar discectomy (TELD) is considered to be an alternative treatment for lumbar disc her- niation requiring surgical treatment. Kambin et al. ${ }^{4)}$ introduced the concept of the "triangular working zone" on the posterolateral corner of the intervertebral disc between the existing root and the traverse root. Since access to the ventral

- Received : February 27, 2020 •Revised : March 30, 2020 •Accepted : April 14, 2020

- Address for reprints : Dae-Hyun Kim

Department of Neurosurgery, Daegu Catholic University Medical Center, College of Medicine, Catholic University of Daegu, 33 Duryugongwon-ro 17-gil, Nam-gu, Daegu 42472, Korea

Tel : +82-53-650-4251, Fax : +82-53-650-4932, E-mail : daehkim@cu.ac.kr, ORCID : https://orcid.org/0000-0003-4716-9551

This is an Open Access article distributed under the terms of the Creative Commons Attribution Non-Commercial License (http://creativecommons.org/licenses/by-nc/4.0) which permits unrestricted non-commercial use, distribution, and reproduction in any medium, provided the original work is properly cited. 
epidural space is necessary for adequate decompression, horizontal methods of approach have been developed by various investigators ${ }^{4,15,19,20)}$. Ruetten et al. ${ }^{15)}$ introduced the extreme lateral access approach for transforaminal endoscopic surgery. However, an increased horizontal angle may increase the risk of penetrating injury to the abdominal cavity and the retroperitoneal organs. Sometimes these injuries may cause complications such as retroperitoneal hematoma, bowel, or kidney injury. Therefore, it is important to determine the appropriate entry point and angle of approach for successful operations.

Some authors have recommended a pre-operative abdominal computed tomography (CT) scan to determine the entry point and the angle of approach ${ }^{15,20)}$. However, the locations of the retroperitoneal organs and the body cavity change depending on the patient's body position. Most CT scans are obtained with the patients in the supine position, while most surgeries are performed with the patient in the prone position. The prone position may result in a downward shift of organ locations relative to the supine position. This downward shift makes a more horizontal surgical approach possible. We hypothesized that if CT scans were obtained from patients in the prone position, similar to surgical positioning, the location of the retroperitoneal space could be determined more accurately.

The purpose of this study was to determine the ideal entry point for transforaminal endoscopic surgery. We verified the safety of conventional methods and examined the ideal entry points and angles of approach at each lumbar spine level using
CT scans obtained from patients in the prone position. We also evaluated the patient characteristics related to the ideal entry point.

\section{MATERIALS AND METHODS}

This study was approved by Institutional Review Board of Daegu Catholic University Medical Center (IRB No. CR-20013). The study was performed using CT scans obtained from patients who visited our clinic for a lumbar spine evaluation between January and December 2018. Thirty-five CT scans from 20 males and 15 females were used for the image analysis. Patients had an average age of 61 years (range, 23-82). Patients who had previous lumbar spine surgery, a gross deformity, or a lumbar vertebral body fracture below the L2 level were excluded.

Images were acquired using a single CT scanner (SOMATOM Definition Flash/WCT-800-140; Siemens AG, Muenchen, Germany). Patients were placed on an operating frame (Wilson's frame) with their abdomens relaxed. Abdominal CT images including lumbar spine, intra-abdominal organs, and skin were then obtained. Axial CT images were reconstructed parallel to each disc space (L2-3, L3-4, and L4-5) at $2 \mathrm{~mm}$ intervals (Fig. 1).

To investigate the risk of retroperitoneal or visceral violations during conventional approach methods for transforami-

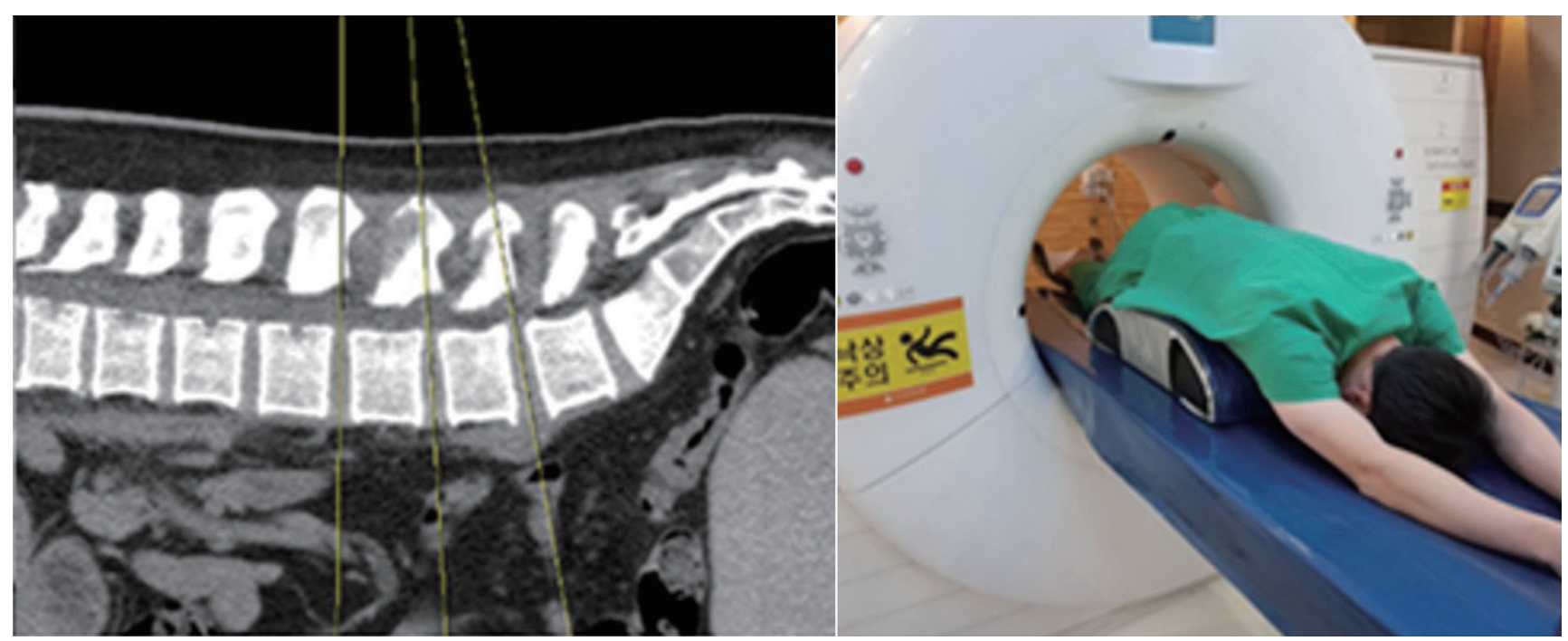

Fig. 1. Patients were positioned on an operating frame (Wilson's Frame) with their abdomens relaxed. Computed tomography images were reconstructed parallel to each disc space (L2-3, L3-4, and L4-5) at $2 \mathrm{~mm}$ intervals. 
nal endoscopic surgery, we identified five conventional approach methods based on previous articles ${ }^{5,13,15,18)}$ and verified them with CT images obtained from patients in the prone position. Five lines of approach were drawn on the axial CT image at each intervertebral disc space level (L2-3, L3-4, and L4-5) using the picture archiving and communication system (PACS).

Kambin and Gellman ${ }^{5)}$ suggested the entry point be located $8-12 \mathrm{~cm}$ away from the midline, while Mayer and Brock ${ }^{13)}$ suggested the entry point should be $9-11 \mathrm{~cm}$ away from the midline. We investigated entry points 10,11 , and $12 \mathrm{~cm}$ away from the midline. On the other hand, Ruetten et al. ${ }^{15)}$ suggested that the inferior limitation of the entry point should be the inferior articular process as observed using lateral X-ray. We refer to this method as the "far lateral approach." There was too much variation in the approach angles and entry point distances between the $12 \mathrm{~cm}$ distance approach and the far lateral approach. We thus decided to also include the "modified far lateral approach," in which the inferior limitation of the entry point was located at the midpoint between the tip of the spinous process and the dorsal edge of the inferior articular process as observed using lateral X-ray. After determining the entry point, a line was drawn to the intersection of the medial pedicular line and the posterior vertebral line. When this line was interrupted by the superior articular process, we drew a tangential line to the ventrolateral border of the superior articular process. The angles of approach were measured, and for every line we screened for retroperitoneal or visceral violations.

Subsequently, the ideal approach lines were drawn on the axial CT image for each level of the intervertebral disc space (L2-3, L3-4, and L4-5) using PACS (Fig. 2). The ideal approach lines were drawn using two methods. In the first method, the starting point was marked on the intersection of the posterior vertebral line and the medial pedicular line. A tangential line was drawn from this point to the posterior margin of the abdominal organs (kidney or bowel). This line was close to horizontal and was used as the boundary line for retroperitoneal organ violations. In the second method, the starting point was marked on the intersection of the posterior vertebral line and the medial pedicular line. A tangential line was drawn from the starting point to the posterior margin of the retroperitoneal membrane. This line was considered the safest approach angle with the lowest risk of retroperitoneal organ violation. The distances of the ideal entry points and angles of approach from the midline and the depth of the posterior vertebral line from the skin were then measured.

The lines above were drawn on both the left and right sides in all 35 patients (70 lines total). All radiological measurements were performed independently by a single experienced
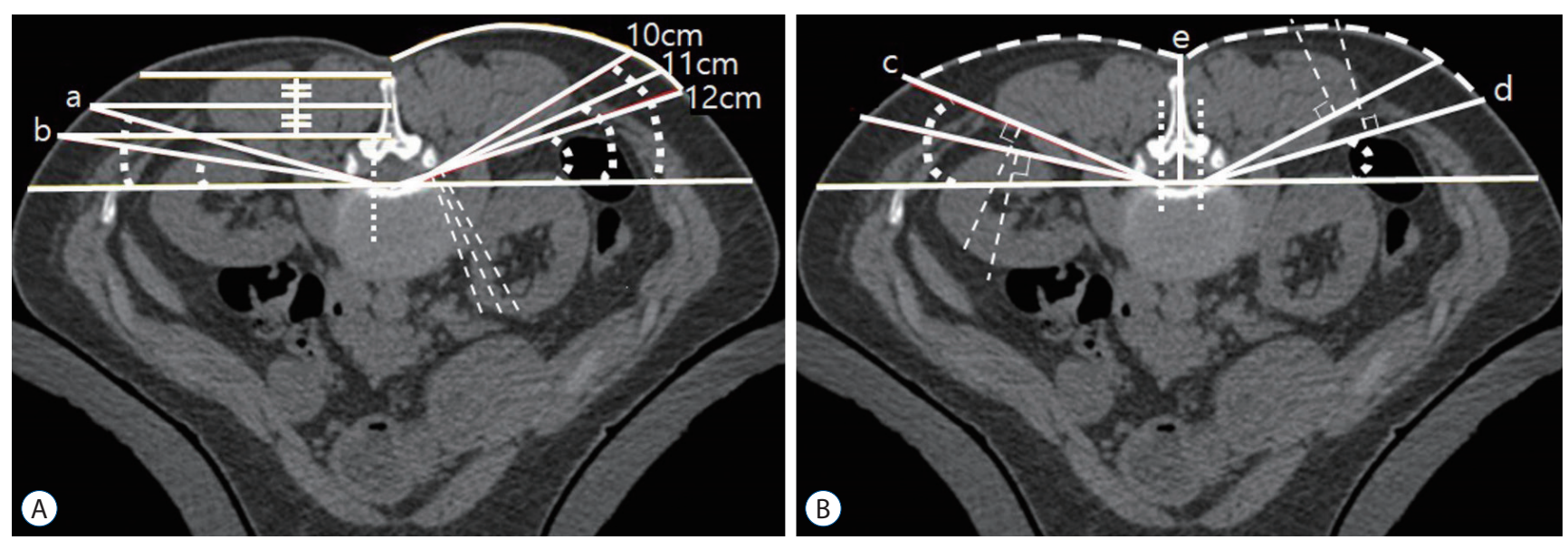

Fig. 2. Five conventional lines of approach (A) and the ideal lines of approach for avoiding visceral or retroperitoneal violations (B). a : Another line is horizontally extended line from the midpoint between the tip of the spinous process and the dorsal edge of the inferior articular process to the intersection of the medial pedicular line and the posterior vertebral line. $b$ : The line from the intersection of the skin and the line horizontally extended from the dorsal edge of the inferior articular process to the intersection of the medial pedicular line and the posterior vertebral line. $c$ : The tangential line from the intersection of the posterior vertebral line and the medial pedicular line to the posterior margin of the retroperitoneum. $d:$ The tangential line from the intersection of the posterior vertebral line and the medial pedicular line to the posterior margin of the abdominal organs. e : The depth of the posterior vertebral line from the skin. 
radiologist who clearly understood the purpose of this study.

Statistical analysis was performed using SPSS Statistics version 23 (IBM Corp., Armonk, NY, USA). Mean values and standard deviations were calculated. The Pearson correlation test was used to determine the correlations between the ideal entry point location and patient factors such as age, weight, height, body mass index (BMI), and the depth of the posterior vertebral line from the skin.

\section{RESULTS}

Thirty-five patients were enrolled in this study. Eighteen patients were diagnosed with lumbar disc herniation with radiculopathy, six patients were diagnosed with lumbar stenosis, and 11 patients underwent CT scans during regular followups for simple compression fracture of the thoracolumbar (T11-L1) segment. Other patient characteristics are summarized in Table 1.

The percentages of retroperitoneal and visceral violations according to the five lines of approach at each level are summarized in Fig. 3. At the L2-3 level, the rates of retroperitoneal violation were high for the $12 \mathrm{~cm}$ distance approach (75\%), the modified far lateral approach (58.3\%), and the far lateral approach (91.7\%). The rate of visceral violation in the far lateral approach was also high (33.3\%), but relatively low (4.2\%) for the $12 \mathrm{~cm}$ distance approach at the L2-3 level. For the L3-4 and the L4-5 levels, rates of retroperitoneal violations were relatively high for the $12 \mathrm{~cm}$ distance approach (L3-4, 37\%; L4-5, 38\% respectively), the modified far lateral approach

Table 1. Patient characteristics

\begin{tabular}{lc}
\hline & Value \\
\hline Sex, male : female & $20: 15$ \\
Age (years) & $60.8 \pm 16.2$ \\
Height $(\mathrm{cm})$ & $163.4 \pm 7.8$ \\
Weight $(\mathrm{kg})$ & $66.3 \pm 13.1$ \\
BMI $\left(\mathrm{kg} / \mathrm{m}^{2}\right)$ & $24.7 \pm 4.3$ \\
L2-3 DPVS $(\mathrm{mm})$ & $57.8 \pm 6.9$ \\
L3-4 DPVS $(\mathrm{mm})$ & $60.4 \pm 7.3$ \\
L4-5 DPVS $(\mathrm{mm})$ & $60.9 \pm 8.0$ \\
\hline
\end{tabular}

Values are presented as mean \pm standard deviation unless otherwise indicated. BMI : body mass index, DPVS : depth of the posterior vertebral line from the skin
(L3-4, 44.3\%; L4-5, 29.2\%), and the far lateral approach (L34, 68.6\%; L4-5, 58.3\%). However, these rates were much lower than those at the L2-3 level. At the L3-4 level, visceral violations were observed relatively infrequently (7.1\%) for the far lateral approach. The rates of visceral violations for the $12 \mathrm{~cm}$ distance and modified far lateral approaches at the L3-4 level were not determined. At the L4-5 level, we observed no visceral violations for any of the approaches.

\section{L2-3}
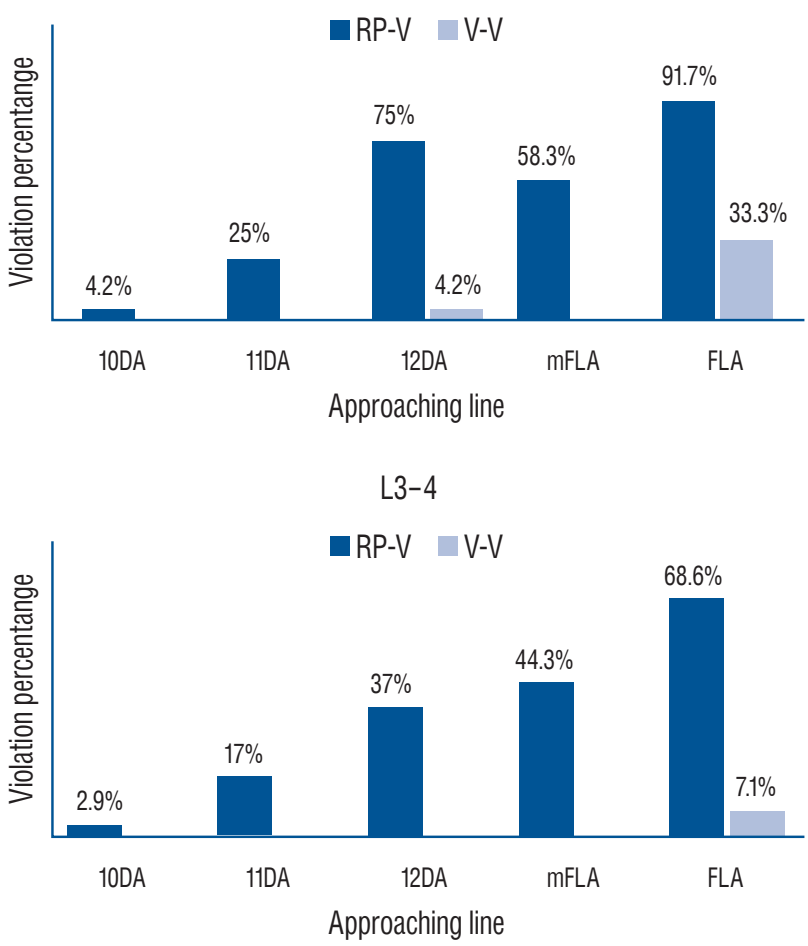

L4-5

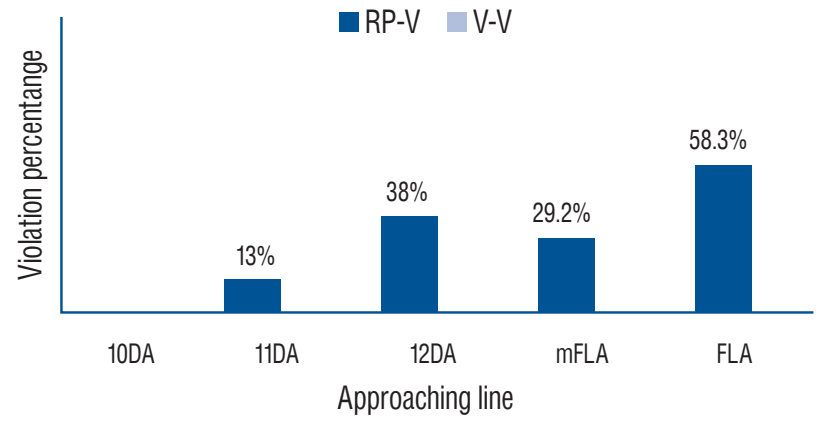

Fig. 3. The percentages of retroperitoneal and visceral violations according to the five lines of approach at each level of the lumbar spine. RP-V : retroperitoneal violation, $\mathrm{V}-\mathrm{V}$ : visceral violation, 10DA : $10 \mathrm{~cm}$ distance approach, 11DA : $11 \mathrm{~cm}$ distance approach, 12DA : $12 \mathrm{~cm}$ distance approach, mFLA : modified far lateral approach, FLA : far lateral approach. 
Table 2. Angles of approach in five methods of approach included in this study

\begin{tabular}{lccc}
\hline & L2-3 & L3-4 & L4-5 \\
\hline 10DA $\left(^{\circ}\right)$ & $25.3 \pm 7.6$ & $26.6 \pm 7.4$ & $27.8 \pm 7.3$ \\
\hline 11DA $\left(^{\circ}\right)$ & $21.0 \pm 7.5$ & $21.7 \pm 7.8$ & $22.9 \pm 7.9$ \\
\hline 12DA $\left(^{\circ}\right)$ & $16.6 \pm 7.4$ & $17.2 \pm 7.9$ & $18.3 \pm 7.9$ \\
$\mathrm{mFLA}\left({ }^{\circ}\right)$ & $17.8 \pm 4.6$ & $17.2 \pm 4.2$ & $17.0 \pm 3.6$ \\
$\mathrm{FLA}\left({ }^{\circ}\right)$ & $10.5 \pm 2.3$ & $10.3 \pm 3.8$ & $8.7 \pm 1.8$ \\
\hline
\end{tabular}

Values are presented as mean \pm standard deviation. 10DA : $10 \mathrm{~cm}$ distance approach, 11DA: $11 \mathrm{~cm}$ distance approach, 12DA: $12 \mathrm{~cm}$ distance approach, mFLA : modified far lateral approach, FLA : far lateral approach

Table 3. The distances from the midline for ideal entry points and the ideal angles of approach for avoiding retroperitoneal violation at each level

\begin{tabular}{lcc}
\hline & Distance $(\mathbf{m m})$ & Angle $\left(^{\circ}\right)$ \\
\hline L2-3 Ideal entry point without RPV & $12.1 \pm 1.4$ & $17.7 \pm 7.6$ \\
L3-4 Ideal entry point without RPV & $13.2 \pm 2.0$ & $15.6 \pm 7.4$ \\
L4-5 Ideal entry point without RPV & $13.9 \pm 1.9$ & $12.0 \pm 6.1$ \\
\hline
\end{tabular}

Values are presented as mean \pm standard deviation. RPV : retroperitoneal violation

The angles of approach for the five methods are summarized in Table 2. Angles of approach for the $10 \mathrm{~cm}$ distance approach were much higher than those for the other methods. In contrast, angles of approach for the far lateral approach were only around $10^{\circ}$. The angles of approach for the 10,11 , and $12 \mathrm{~cm}$ distance approaches increased moving caudally along the spine, while the angles of approach for the modified far lateral and the far lateral approaches decreased moving caudally along the spine.

The distances of the ideal entry points to the midline and the ideal angles of approach for avoiding retroperitoneal violation for each level are summarized in Table 3. Ideal entry point tended to be far from the midline, and ideal angles of approach tended to become more horizontal as one moved from the upper levels of the lumbar spine to the lower ones.

The ideal angles of approach for avoiding retroperitoneal and visceral violations at each level are summarized in Table 4. When we measured the ideal angles of approach for avoiding visceral violations, angles with negative values were not analyzed since such approaches are not practical. Only 29 (15 right and 14 left) lines had positive values at the L2-3 level, 10
Table 4. The ideal angles of approach for avoiding retroperitoneal or visceral violations at each level

\begin{tabular}{lcc}
\hline & IA RPV & IA V V \\
\hline Right $\left(^{\circ}\right)$ & & \\
$\operatorname{L} 2-3(n=15)$ & $21.8 \pm 4.9$ & $8.3 \pm 6.2$ \\
$\operatorname{L3}-4(n=6)$ & $21.7 \pm 6.6$ & $9.0 \pm 2.0$ \\
$\operatorname{L4}-5(n=1)$ & 14 & 4 \\
Left $\left(^{\circ}\right)$ & & \\
$\operatorname{L}-3(n=14)$ & $21.4 \pm 6.2$ & $9.9 \pm 5.7$ \\
$\operatorname{L3}-4(n=4)$ & $21.8 \pm 7.1$ & $11.2 \pm 5.9$ \\
$\operatorname{L} 4-5(n=1)$ & 11 & 3 \\
\hline
\end{tabular}

Values are presented as mean \pm standard deviation or mean. IA : ideal angle, RPV : retroperitoneal violation, $\mathrm{VV}$ : visceral violation

lines (six right and four left) had positive values at the L3-4 level, and two lines (one right and one left) had positive values at the L4-5 level.

The Pearson correlation test was used to determine the correlations between the distance of the ideal entry points to the midline, the ideal angles of approach, and patient characteristics such as age, height, BMI, weight, and the depth of the posterior vertebral line from the skin. BMI, weight, and the depth of the posterior vertebral line from the skin were positively and significantly correlated with the distance of the ideal entry points to the midline. We observed no other significant relationships between these variables (Fig. 4).

\section{DISCUSSION}

As life expectancy has increased, the age of patients with spinal diseases has also increased. Endoscopic surgery is advantageous in such patients, as it results in minimal muscle and bone damage, less pain, quicker rehabilitation, and reduced duration of hospitalization. With the development of surgical tools and techniques, endoscopic spinal surgery has expanded to include other aspects of spinal diseases, resulting in good clinical outcomes ${ }^{8)}$. TELD belongs to the first generation of endoscopic spinal surgery techniques. There are numerous articles about this technique, and recent studies report that the clinical results of transforaminal endoscopy are similar to traditional open surgery ${ }^{9,11,14)}$.

The goal of TELD is to remove the herniated disc with minimal damage to the musculoskeletal structure. Success depends 

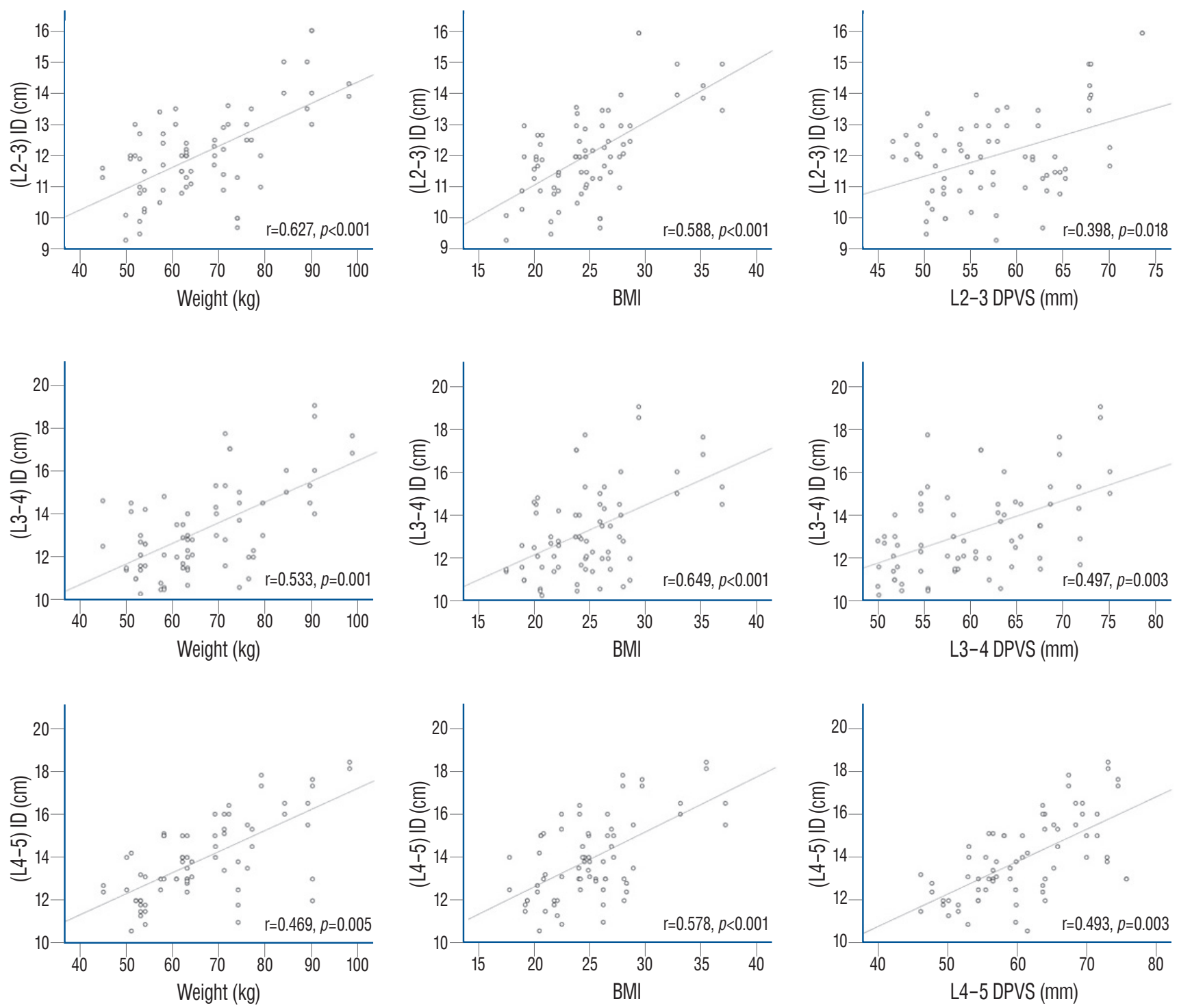

Fig. 4. Scatter graphs for the correlation between the distance of the entry point and body mass index (BMI), weight, and the depth of the posterior vertebral line from the skin (DPVS) at the L2-3, the L3-4, and L4-5 levels.

on the proper placement of the endoscope at the posterior intervertebral disc. However, endoscope placement involves several obstacles such as the superior articular process of the facet or narrow access to the "triangular working zone," increasing the risk of nerve root injury. TELD techniques have continuously evolved, resulting in approaches such as the outside in (Schubert and Hoogland ${ }^{16)}$ ), the inside out (Yeung and Yeung ${ }^{20)}$ ), and the mobile outside in techniques".

On the other hand, a more horizontal approach would allow easier access to the "triangular working zone," greater direct visualization of the protruded disc and the epidural space, and more convenient manipulation of surgical instruments.
Because the tip of the endoscopic device is fixed to the "triangular working zone," a horizontal approach requires a more distant entry point for adequate decompression. A more distant entry point may therefore enable a more horizontal approach.

Kambin and Gellman ${ }^{5}$ suggested the appropriate angle of approach could be $35-45^{\circ}$ and the entry point could be located $8-12 \mathrm{~cm}$ from the midline. Mayer and Brock ${ }^{13)}$ suggested the angle of approach could be $30-40^{\circ}$ and the entry point could be located $9-11 \mathrm{~cm}$ from the midline. Yeung ${ }^{18)}$ suggested the appropriate angle of approach could be $25-30^{\circ}$ and the average distance from the midline was $11 \mathrm{~cm}$. Stücker ${ }^{17)}$ suggest- 
ed the entry point distance should be less than $12 \mathrm{~cm}$ for patients shorter than $170 \mathrm{~cm}$, more than $14 \mathrm{~cm}$ for patients taller than $180 \mathrm{~cm}$, and less than $12 \mathrm{~cm}$ for extremely thin patients. They also suggested that the angle of approach should be above $20^{\circ}$. Several researchers have proposed a more horizontal approach. For example, Ruetten et al. ${ }^{15)}$ introduced the extreme lateral approach. Lee et al. ${ }^{10)}$ reported that a true lateral approach for TELD would be possible if the patient was in the prone position, due to the ventral shift of the abdominal organs. However, the far lateral approach may increase the risk of retroperitoneal or visceral violations. Injury to the bowel or the large vessels would be extremely rare but potentially disastrous complications. Immediate and aggressive management of these complications is harder during endoscopic surgery compared to open surgery. The risk of retroperitoneal and visceral violations has still not been evaluated adequately in previous studies, despite the increasing clinical use of increasingly horizontal angles of approach.

Despite the relatively high rates of retroperitoneal violation during the conventional methods in our study, the likelihood of complications such as retroperitoneal organ injury would be extremely low. Various studies have described a variety of complications caused by endoscopic lumbar surgery such as dural tear, incomplete decompression, neural injury, and retroperitoneal hemorrhage due to injury to the segmental lumbar arteries ${ }^{1,2,6,12)}$. Matsumoto et al. ${ }^{12)}$ reported that out of 6239 spinal endoscopic surgeries, 133 had complications after endoscopic lumbar surgery, but none of these complications were related to visceral violations. This finding may be explained by the significant differences we observed between the ideal angle of approach for avoiding retroperitoneal violations and the ideal angle of approach for avoiding visceral violations. In other words, the ideal line of approach for avoiding visceral violations is relatively safe. However, when it comes to absolute safety, the ideal line of approach for avoiding retroperitoneal violation is likely more suitable. Hence, the incidence of retroperitoneal violations would be a great measure of overall safety.

Our results suggest that the number of retroperitoneal violations was significantly lower at caudal locations relative to rostral ones. A more horizontal approach may therefore be safer at lower levels of the lumbar spine. However, at the L2-3 level we observed high rates of visceral violation during horizontal approaches. Ruetten et al. ${ }^{15)}$ reported that a less lateral access point could be used at the L1-2 and the L2-3 levels due to the enlargement of the intervertebral foramen toward the cranial end. Ahn ${ }^{1)}$ also recommended a steeper approach for the L1-2 and the L2-3 levels due to a high risk of nerve root injury during the horizontal approach. Therefore, we recommend a less horizontal approach at the L2-3 level. We also suggest using an entry point located less than $10 \mathrm{~cm}$ from the midline at the L2-3 level is extremely safe. However, Hurday et al. ${ }^{3)}$ analyzed 100 magnetic resonance images and concluded that as foraminal width decreases caudally, the nerve root at more caudal positions lies in a more ventral position to the disc. Such a trajectory angle at more caudal locations would thus be too narrow or steep ${ }^{3)}$. A more horizontal approach is therefore required at the L3-4 and the L4-5 levels. According to our results, the ideal angles of approach for avoiding retroperitoneal violation were much lower than the expected values. These results may be helpful for surgeons who still hesitate to use the extremely horizontal approach during TELD. However, for an extremely safe approach at the L3-4 and the L4-5 levels, we recommend marking the inferior entry point limitation at the upper one-third portion between the tip of the spinous process and the dorsal edge of the inferior articular process as observed using lateral X-ray. We base this recommendation on the high rates of retroperitoneal violation we observed during the modified far lateral approach.

Various authors have suggested that obese patients might require a more distant entry point from the midline ${ }^{5,13,15-17)}$. In this study, we examined characteristics such as age, height, weight, BMI, and the depth of the posterior vertebral line from the skin. With correlation analysis, we observed that both BMI and the depth of the posterior vertebral line from the skin positively correlated with the ideal entry point distance from the midline. These results indicate that we should consider the patient's body shape while determining the entry point for TELD.

At more caudal levels, the ideal entry point tended to be more distant from the midline and the ideal angle of approach was more horizontal relative to more rostral levels. This finding may be related to the position of the patients. Since patients were on a Wilson frame, the L4-5 level was located at the highest position. This positioning would cause increased downward migration of the abdominal organs at the L4-5 level. The more caudal retroperitoneal organs also tend to be under the posterior vertebral line. This finding is mainly re- 
lated to the existence of the kidney at the L2-3 level. However, this trend could also be related to the positioning of patients. One limitation of our study is that it does not differentiate between these possibilities, which would require comparisons with supine CT images. Another limitation of this study is that the results are inapplicable to surgeries performed on other surgical frames such as the Jackson table, as such frames may have a different effect on retroperitoneal organ and cavity location compared to the Wilson's frame. Moreover, the preoperative CT scans may not perfectly mimic surgical conditions. In the future, intraoperative CT scanning and navigation systems may play an important role in determining the ideal entry point and angle of approach during TELD.

\section{CONCLUSION}

Our study investigated the ideal entry point and angle of approach during TELD. Based on CT images obtained from patients in the prone position, we reviewed the risk of retroperitoneal and visceral violations during extreme lateral approach and other well-known methods of approach. Based on our observations and previous results, we suggest an ideal entry point at each level of the lumbar spine. We also observed a positive correlation between the entry point distance and patient characteristics such as BMI, weight, and the depth of the posterior vertebral line from the skin.

\section{CONFLICTS OF INTEREST}

No potential conflict of interest relevant to this article was reported.

\section{INFORMED CONSENT}

This type of study does not require informed consent.

\section{AUTHOR CONTRIBUTIONS}

\author{
Conceptualization : JUL, DHK \\ Data curation : JUL, KJP
}

\author{
Formal analysis : JUL, DHK, YHL \\ Funding acquisition : JUL, DHK, MKC, KHK \\ Methodology : JUL, DHK, KHK, MKC, KJP \\ Project administration : JUL, DHK, MKC \\ Visualization : JUL, DHK \\ Writing - original draft : JUL, DHK \\ Writing - review \& editing : JUL, DHK, MKC
}

\section{ORCID}

$\begin{array}{ll}\text { Jong Un Lee } & \text { https://orcid.org/0000-0002-1617-7789 } \\ \text { Ki Jeoung Park } & \text { https://orcid.org/0000-0001-7756-2632 } \\ \text { Ki Hong Kim } & \text { https://orcid.org/0000-0002-2491-2261 } \\ \text { Man Kyu Choi } & \text { https://orcid.org/0000-0001-6670-7541 } \\ \text { Young Hwan Lee } & \text { https://orcid.org/0000-0002-5535-2906 } \\ \text { Dae-Hyun Kim } & \text { https://orcid.org/0000-0003-4716-9551 }\end{array}$

\section{References}

1. Ahn Y : Transforaminal percutaneous endoscopic lumbar discectomy: technical tips to prevent complications. Expert Rev Med Devices 9 : 361-366, 2012

2. Ahn Y, Kim JU, Lee BH, Lee SH, Park JD, Hong DH, et al. : Postoperative retroperitoneal hematoma following transforaminal percutaneous endoscopic lumbar discectomy. J Neurosurg Spine 10 : 595-602, 2009

3. Hurday Y, Xu B, Guo L, Cao Y, Wan Y, Jiang H, et al. : Radiographic measurement for transforaminal percutaneous endoscopic approach (PELD). Eur Spine J 26 : 635-645, 2017

4. Kambin P, Casey K, O'Brien E, Zhou L : Transforaminal arthroscopic decompression of lateral recess stenosis. J Neurosurg 84 : 462-467, 1996

5. Kambin P, Gellman $H$ : Percutaneous lateral discectomy of the lumbar spine a preliminary report. Clin Orthop Relat Res 174 : 127-132, 1983

6. Kamson S, Trescot AM, Sampson PD, Zhang Y : Full-endoscopic assisted lumbar decompressive surgery performed in an outpatient, ambulatory facility: report of 5 years of complications and risk factors. Pain Physician 20 : E221-E231, 2017

7. Kim HS, Adsul N, Kapoor A, Choi SH, Kim JH, Kim KJ, et al. : A mobile outside-in technique of transforaminal lumbar endoscopy for lumbar disc herniations. J Vis Ex (138) : 57999, 2018

8. Kim M, Kim HS, Oh SW, Adsul NM, Singh R, Kashlan ON, et al. : Evolution of spinal endoscopic surgery. Neurospine $16: 6-14,2019$

9. Kim M, Lee S, Kim HS, Park S, Shim SY, Lim DJ : A comparison of percutaneous endoscopic lumbar discectomy and open lumbar microdiscectomy for lumbar disc herniation in the Korean: a meta-analysis. Biomed 
Res Int 2018 : 9073460, 2018

10. Lee DH, Kim NH, Park JB, Hwang CJ, Lee CS, Kim YT, et al. : CT scan assessment of the pathway of the true lateral approach for transforaminal endoscopic lumbar discectomy: is it possible? J Bone Joint Surg Br 93 : 1395-1399, 2011

11. Li X, Han Y, Di Z, Cui J, Pan J, Yang M, et al. : Percutaneous endoscopic lumbar discectomy for lumbar disc herniation. J Clin Neurosci 33 : 1927, 2016

12. Matsumoto M, Hasegawa $T$, Ito M, Aizawa T, Konno S, Yamagata M, et al. : Incidence of complications associated with spinal endoscopic surgery: nationwide survey in 2007 by the Committee on Spinal Endoscopic Surgical Skill Qualification of Japanese Orthopaedic Association. J Orthop Sci 15 : 92-96, 2010

13. Mayer HM, Brock M : Percutaneous endoscopic discectomy: surgical technique and preliminary results compared to microsurgical discectomy. J Neurosurg 78 : 216-225, 1993

14. Ruan W, Feng F, Liu Z, Xie J, Cai L, Ping A : Comparison of percutaneous endoscopic lumbar discectomy versus open lumbar microdiscectomy for lumbar disc herniation: a meta-analysis. Int J Surg 31 : 86-92, 2016

15. Ruetten S, Komp M, Godolias G : An extreme lateral access for the surgery of lumbar disc herniations inside the spinal canal using the fullendoscopic uniportal transforaminal approach-technique and prospective results of 463 patients. Spine (Phila Pa 1976) 30 : 2570-2578, 2005

16. Schubert $M$, Hoogland $T$ : Endoscopic transforaminal nucleotomy with foraminoplasty for lumbar disk herniation. Oper Orthop Traumatol $17:$ 641-661, 2005

17. Stücker R : The Transforaminal Endoscopic Approach in Mayer HM (ed) : Minimally Invasive Spine Surgery: A Surgical Manual. Berlin : Springer Berlin Heidelberg, 2000, pp201-205

18. Yeung AT : The evolution and advancement of endoscopic foraminal surgery: one surgeon's experience incorporating adjunctive techologies. SAS J 1 : 108-117, 2007

19. Yeung AT, Tsou PM : Posterolateral endoscopic excision for lumbar disc herniation: surgical technique, outcome, and complications in 307 consecutive cases. Spine (Phila Pa 1976) 27 : 722-731, 2002

20. Yeung AT, Yeung CA : Minimally invasive techniques for the management of lumbar disc herniation. Orthop Clin North Am 38 : 363-372; abstract vi, 2007 